\title{
Draft Genome Sequences of Proteus mirabilis K1609 and K670: A Model Strains for Territoriality Examination
}

\author{
Dawid Gmiter $^{1}$ (D) $\cdot$ Grzegorz Czerwonka $^{1} \cdot$ Justyna Malgorzata Drewnowska ${ }^{2}$ Izabela Swiecicka $^{2,3} \cdot$ Wieslaw Kaca $^{1}$
}

Received: 18 April 2018 / Accepted: 12 November 2018 / Published online: 17 November 2018

(c) The Author(s) 2018

\begin{abstract}
Proteus mirabilis is a pathogenic Gram-negative bacterium characterized by its ability to swarm across surfaces, which frequently leads to colonization of the urinary tract and causes severe infections. P. mirabilis strains are also well known from their self-recognition phenomenon, referred to as Dienes phenomenon. In this study, we present novel aspect of selfrecognition, which is a hierarchy in terms of strains territoriality. We report the draft genome sequences of $P$. mirabilis K1609 and K670 strains exhibiting the strongest and the weakest territoriality, respectively. Our results indicated that K1609 is closely related to strain BB2000, a model system for self-recognition, comparing with the K670. We annotated genes associated with recognition of kin and swarming initiation control and indicated polymorphisms by which observed differences in territoriality might results from. The phenotypic and genomic features of both strains reveal their application as a model organisms for studying not only the mechanisms of kin-recognition but also strains territoriality, thus providing new approach to the phenomenon. Availability of these genome sequences may facilitate understanding of the interactions between P. mirabilis strains.
\end{abstract}

\section{Introduction}

Proteus mirabilis is a Gram-negative urinary tract pathogen that exhibits remarkable ability to swarm over the solid surface. Swarming motility is a complex social behavior requiring cell to cell communication, and possible virulence factor allowing $P$. mirabilis to gain access to the bladder by migration along the external surfaces of the catheter [1]. $P$. mirabilis is also well known from its territorial behavior, which manifests in form of the demarcation line formation at the edge of approaching swarms. The phenomenon, referred to as Dienes phenomenon, is known for over a five decades [2]. Demarcation line occurs between non-kin strains and the process is governed by the action of type VI secretion

Dawid Gmiter

dawid.gmiter@ujk.edu.pl

1 Department of Microbiology, Jan Kochanowski University, 15 Swietokrzyska Street, 25-406 Kielce, Poland

2 Departament of Microbiology, Institute of Biology, University of Bialystok, 1J Ciolkowskiego Street, 15-245 Bialystok, Poland

3 Laboratory of Applied Microbiology, University of Bialystok, 1J Ciolkowskiego Street, 15-245 Bialystok, Poland system (TVISS) exporting proteins that determinate strains kin recognition $[3,4]$. The previous studies indicated the role of $i d s A B C D E F$ and $i d r A B C D E$ [4] as well as primary $h p c$-vgrG effector (pef) operons $[3,5]$ in the phenomenon; however, the exact mechanism is poorly understood.

To date, only $P$. mirabilis HI4320 and P. mirabilis BB2000 were completely sequenced $[6,7]$ as an examples of strains employed in self-recognition and competition examination $[5,8]$. The BB2000 strain was first in which the self-recognition genes were identified [9]. Here, we present novel aspect of self-recognition in P. mirabilis, which is a hierarchy in terms of strains territoriality, and report genome sequences of $P$. mirabilis $\mathrm{K} 1609$ and K670 strains. Both strains have been chosen because of their remarkably differences in territorial advantages on solid surface. Based on phenotypic and genomic differences, we proposed these strains as a model organisms for territoriality examination. Our two-strains system is unique comparing to the previously used. It allows for a thorough investigation focused on the mechanisms of territoriality among $P$. mirabilis, which contributes to a better understanding of the phenomenon. 


\section{Materials and Methods}

\section{Strains, Genome Sequences, and Territoriality Assay}

The five $P$. mirabilis strains used in this study are presented in Table 1. Study included three clinical isolates from the Holly Cross Cancer Center in Kielce and two laboratory strains obtained from the Czech National Collection of Type Cultures in Prague, Czech Republic. Following the matrix laser desorption ionization-time of flight mass spectrometry (MALDI-TOF MS) spectrum analysis, strains were deposited in Polish Collection of Microorganisms of the Ludwik Hirszfeld Institute of Immunology and Experimental Therapy, Polish Academy of Science, Wroclaw. Strains were maintained in Lysogenic broth (LB) (Biocorp, Poland) supplemented with 8\% DMSO at the temperature of $-80^{\circ} \mathrm{C}$. Used genome sequences of $P$. mirabilis strains are presented in Table 2. The LB was used for culturing, and LB plates with the 1.5\% agar (Biocorp, Poland) were used for swarming experiments—swarming agar. For the territoriality examination, the $2.5 \mu \mathrm{l}$ of strains suspensions (1:100 dilution of overnight culture) were spotted in opposition onto the swarming agar plates and allowed to swarm for $18 \mathrm{~h}$ at $37{ }^{\circ} \mathrm{C}$.

\section{Sequencing}

Genomic DNA was isolated using the QIAamp DNA Micro Kit (Qiagen GmbH, Hilden, Germany) according to the manufacturer's procedure with a protocol for Gram-negative bacteria. Final elution was performed with nuclease-free water. DNA quality was assessed using a NanoDrop 2000 Spectrophotometer (Thermo Fisher Scientific, Wilmington, USA). The quantity was measured using both the Qubit 2.0 Fluorometer with Qubit dSDNA HS Assay Kit (Invitrogen, Thermo Fisher Scientific, Wilmington, USA) and the 2200 TapeStation Instrument with Genomic DNA ScreenTape Assay (Agilent Technologies Inc., St Clara, CA, USA). Libraries were prepared using the Nextera XT kit (Illumina Inc., San Diego, CA, USA) according to the manufacturer's protocol and quantified by capillary electrophoresis applying
Table 1 Strains used in the study
Table 2 Complete and draft genome sequences of Proteus mirabilis strains used in this study

\begin{tabular}{llc}
\hline Strain & Reference and source & \#PCM* \\
\hline K1609 & This study; Holly Cross Cancer Center in Kielce, Poland & 2877 \\
K670 & {$[10] ;$ Holly Cross Cancer Center in Kielce, Poland } & 2871 \\
K12796 & This study; Holly Cross Cancer Center in Kielce, Poland & 2866 \\
PrK 34/57 & [10]; Czech National Collection of Type Cultures in Prague, Czech Republic & 2874 \\
PrK 61/57 & [10]; Czech National Collection of Type Cultures in Prague, Czech Republic & 2875 \\
\hline
\end{tabular}

*\#PCM Deposition number in Polish Collection of Microorganisms

\begin{tabular}{lllll}
\hline Strain & Accession number & Genome size (bp) & Data type & Reference \\
\hline BB2000 & CP004022 & $3,846,754$ & Complete & {$[7]$} \\
HI4320 & AM942759 & $4,063,606$ & Complete & {$[6]$} \\
GN2 & CP026581 & $4,012,640$ & Complete & {$[11]$} \\
1230_SSON & NZ_JVXV01000000 & $3,923,692$ & WGS & {$[12]$} \\
AOUC-001 & CP015347 & $4,272,433$ & Complete & {$[13]$} \\
AR_0029 & CP029725 & $3,980,098$ & Complete & \\
AR_0155 & CP021694 & $4,372,742$ & Complete & \\
AR_0159 & CP021550 & $4,055,152$ & Complete & \\
AR379 & CP029133 & $4,219,380$ & Complete & {$[14]$} \\
ATCC 7002 & NZ_JOVJ00000000 & $3,992,612$ & WGS & {$[15]$} \\
BC11-24 & CP026571 & $4,021,165$ & Complete & {$[16]$} \\
CYPM1 & CP012674 & $3,793,000$ & Complete & \\
PM_125 & NZ_LWUL00000000 & $3,955,474$ & WGS & {$[17]$} \\
PM_178 & NZ_LWUM00000000 & $3,969,065$ & WGS & {$[17]$} \\
Pr2921 & LGTA00000000 & $3,924,499$ & WGS & {$[18]$} \\
T18 & CP017085 & $4,131,426$ & Complete & \\
WGLW4 & NZ_AMGU00000000 & $3,920,397$ & WGS & \\
ATCC 29906 & NZ_ACLE01000000 & $3,975,048$ & WGS & \\
\hline
\end{tabular}


the Agilent High Sensitivity D5000 ScreenTape System (Agilent Technologies Inc.). Libraries were sequenced on the MiSeq machine (Illumina) using v2 reagents with $2 \times 250$ bp paired-end reads. Consequently, 90.2 and $82.4 \%$ of bases of sequencing reads had quality scores $\geq Q 30$ for K1609 and K670, respectively. De novo genome assembly was performed using CLC Genomic Workbench v5 (Qiagen). Plasmid DNA was isolated using AccuPrep Plasmid Mini Extraction Kit (Bioneer Company, Daejeon, South Korea) according to the manufacturer's procedure.

\section{Bioinformatics}

Genome sequences were functionally annotated by Rapid Annotation Subsystems Technology (RAST) server [19] using the ClassicRAST annotation scheme, FIGfams version 90, automatic error correction, and automatic frame shift correction. The genetic relationships of strains were presented using average nucleotide identity (ANI) calculator (http://enve-omics.ce.gatech.edu/ani/index) [20]. The phylogenomic tree was obtained using T-Rex (http://www.trex. uqam.ca/) employing Neighbor-joining method [21]. The genes associated with strains territoriality were annotated manually using BLAST 2.8.0 [22]. For genomes' visual comparison Mauve software was used [23].

\section{Results and Discussion}

\section{Strains Territoriality}

Two $P$. mirabilis strains inoculated on the agar plate start to migrate toward each other. When migrating swarms meet, the formation of Dienes line occurs, if both belong to different Dienes compatibility groups [24]. Our observation allowed to point out that strains display different ability to space occupying. Therefore, we referred territoriality among P. mirabilis as ability of two non-kin swarms to occupy the surface of agar plate in presence of each other.

All five strains submitted to territoriality assay formed Dienes line with each other. The territorial behavior of strains is presented in Fig. 1. In all used combinations, the K1609 tended to occupy larger area of plate comparing to second strain-we defined this as strong territorial advantages. Isolate K12796 and laboratory strain PrK 61/57 exhibited moderate territoriality. The K12796 and PrK 61/57 growth was restricted by K1609 to some extent. Territory of strains K670 and PrK 34/57 was restricted at the highest level in the presence of other competitors so we define this as the weakest territorial advantages. The restriction effect was not so intense for PrK 34/57 comparing with the K670. Strain K1609 restricted the K670 growth at the highest level comparing to the restriction caused by the remaining strains.
Thus, strains K1609 and K670 exhibit the strongest and the weakest territoriality, respectively.

Taking account the observation above, it could be stated that among used $P$. mirabilis strains the hierarchy in terms of territoriality occurs. To our best knowledge the results obtained in this study is the first report of such hierarchy in P. mirabilis territoriality. Previously, it was said that colonization of the plate is largely determined by the rate and initiation of swarming [24]. However, the mechanisms governing that process and its eventual biological importance remain unexplained.

\section{Genomes Characterization and Phylogenomic}

Based on the observation above, K1609 and K670 were selected for DNA sequencing. The main features of strains genomes are presented in Table 3. Both strains possess quite similar genomes size and a number of predicted coding sequences. The distribution of subsystems in K1609 and K670 is presented in Table 4. Only in K1609 we observed the presence of one plasmid, which was not sequenced separately.

Phylogenomic analysis based on whole-genome ANI distance matrix revealed that $P$. mirabilis genomes clustering into two major clades (Fig. 2). We referred these clades as BB2000-like and HI4320-like. Both our studied strains are located in BB2000-like group; however, K1609 is closely related to BB2000 comparing with the K670. The divergence into two clades represented by BB2000 and HI4320 was previously shown by the phylogenetic analysis using 16S rRNA gene [25]. Genome of K1609 clusters with the BB2000, BC11-24 and GN2, meanwhile K670 clusters with the AR_0029, AR379 and AR_0155. Previously it was shown that BB2000 and HI4320 strains exhibit genetic variations corresponding to self-recognition differences [7], which is in line with our observation. Visual comparison of K1609 and K670 with BB2000, HI4320 and their closest relatives is presented in Fig. 3.

\section{Annotation of Genes Potentially Involved in Strains Territoriality}

Our hypothesis assumed that strains exhibiting strong territoriality start migration earlier, which allows to colonize of larger area of plate than it is possible for weak competitors at the same time. After the non-kin swarms contact, spreading of weaker competitor is restricted through the self-recognition mechanisms [3, 4]. At this point, the crucial factors involved in strains territoriality seem to be the migration initiation control and self-recognition mechanisms.

We decided to annotate genes associated with the selfrecognition in P. mirabilis. The RAST annotation predicted in K1609 and K670 genomes the presence of genes encoding 


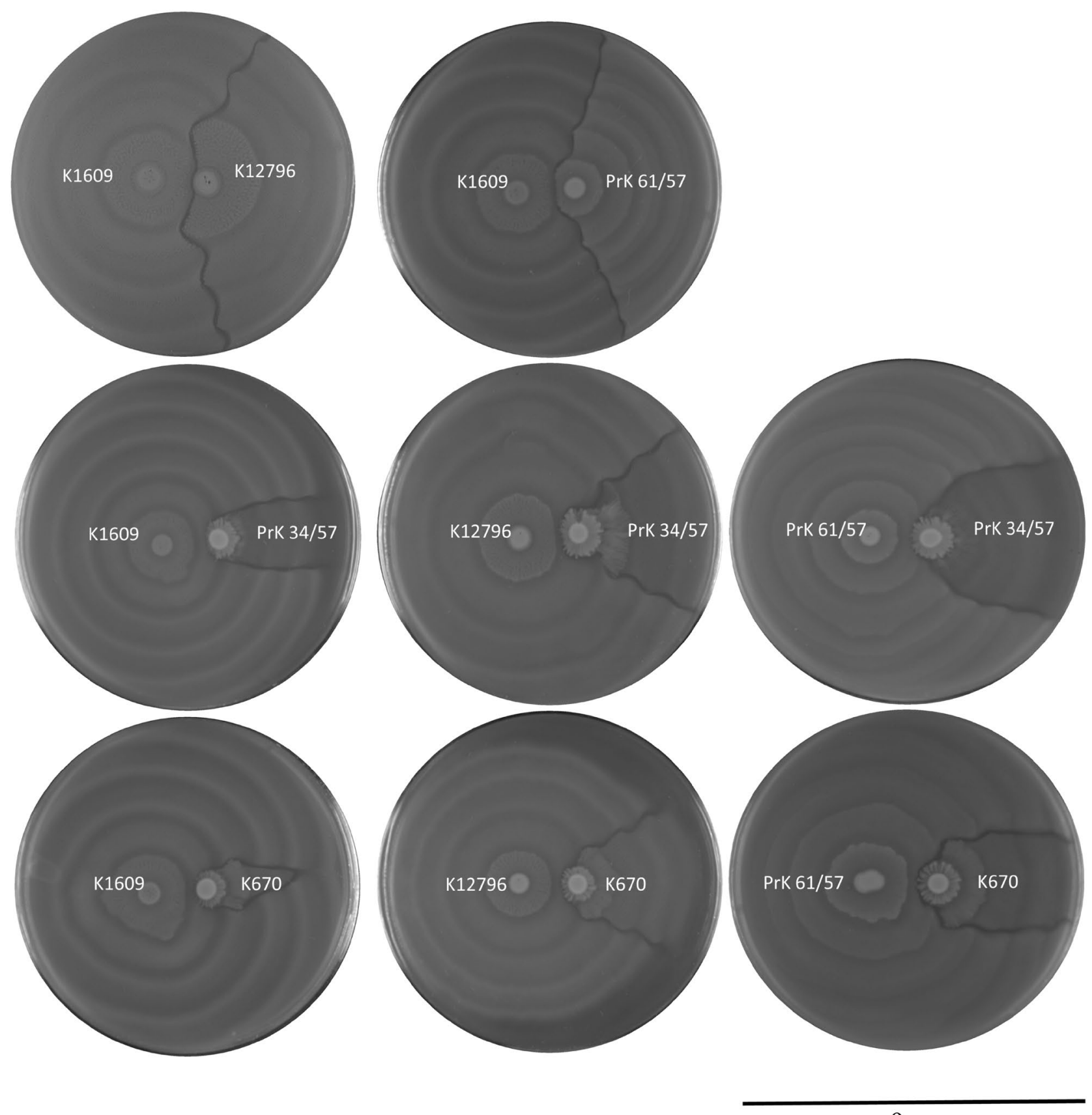

$9 \mathrm{~cm}$

Fig. 1 Territoriality of studied Proteus mirabilis strains. P. mirabilis strains exhibit hierarchy in terms of their territorial advantages. Territoriality is defined as the area of surface occupied by particular

Hpc an VgrG proteins, which are the structural elements of TVISS machinery [26]. Using BLAST comparative analysis, we confirmed the presence of putative TVISS gene locus in both strains. This putative TVISS locus is highly conserved (99\% of homology) with the previously described in BB2000 and HI4320 strains [3, 4].

As the P. mirabilis BB2000 was the first strain in which selfrecognition genes were identified [9], we decided to annotate swarm in presence of non-kin competitor. Strains K1609 and K670 demonstrate the strongest and the weakest territoriality among studied strains, respectively

$i d s$ and $i d r$ operons in K1609 and K670 through manually comparison using BLAST algorithm. Comparison of ids and $i d r$ genes between BB2000, K1609, and $\mathrm{K} 670$ is presented in Fig. 4. The BLAST analysis revealed that K670 strain lacks the ids operon, meanwhile these genes are present in the genome of K1609. The lack of $i d s$ operon in K670 is intrigued considering the role of this operon in $P$. mirabilis self-recognition [27]. However, our screening additionally revealed absence of 
Table 3 Genomes assembly statistics

\begin{tabular}{lll}
\hline Attribute & Value \\
\cline { 2 - 3 } & K1609 & K670 \\
\hline Genome size (bp) & $3,817,795$ & $3,935,626$ \\
\%GC & 38.5 & 38.7 \\
N50 (bp) & 95,718 & 105,852 \\
L50 (bp) & 13 & 13 \\
Number of contigs (with PEGs) & 83 & 76 \\
Number of subsystems & 496 & 496 \\
Number of coding sequences & 3455 & 3568 \\
Number of RNAs & 78 & 82 \\
Number of plasmids & 1 & 0 \\
\hline
\end{tabular}

Table 4 Subsystems distribution of Proteus mirabilis K1609 and K670 strains based on RAST annotation server

\begin{tabular}{|c|c|c|}
\hline Subsystems & K1609 & K670 \\
\hline Cofactors, vitamins, prosthetic groups, pigments & 248 & 248 \\
\hline Cell wall and capsule & 159 & 160 \\
\hline Virulence, disease, and defence & 71 & 73 \\
\hline Potassium metabolism & 25 & 25 \\
\hline Photosynthesis & 0 & 0 \\
\hline Miscellaneous & 43 & 43 \\
\hline Phages, prophages, transposable elements, plasmids & 25 & 23 \\
\hline Membrane transport & 182 & 180 \\
\hline Iron acquisition and metabolism & 55 & 58 \\
\hline RNA metabolism & 223 & 223 \\
\hline Nucleosides and nucleotides & 98 & 97 \\
\hline Protein metabolism & 269 & 275 \\
\hline Cell division and cell cycle & 37 & 36 \\
\hline Motility and chemotaxis & 57 & 57 \\
\hline Regulation and cell signaling & 97 & 97 \\
\hline Secondary metabolism & 4 & 4 \\
\hline DNA metabolism & 104 & 96 \\
\hline Fatty acids, lipids, and isoprenoids & 109 & 108 \\
\hline Nitrogen metabolism & 26 & 26 \\
\hline Dormancy and sporulation & 6 & 6 \\
\hline Respiration & 150 & 151 \\
\hline Stress response & 134 & 134 \\
\hline Metabolism of aromatic compounds & 3 & 3 \\
\hline Amino acids and derivatives & 381 & 366 \\
\hline Sulfur metabolism & 36 & 17 \\
\hline Phosphorus metabolism & 35 & 37 \\
\hline Carbohydrates & 332 & 331 \\
\hline
\end{tabular}

ids operon in complete genomes of AR_0195 and 1230-SSON strains and draft genome of WLGW4. This observation is interesting considering the phylogenomic analysis presented in Fig. 2. It could be seen that only WGLW4 belongs to the same clade as K670 in opposition to AR_0195 and 1230-SSON, which are located in HI4320-like clade.

We observed as well that $i d s E F$ genes are at least duplicated in K1609 strain, which in fact is not precedence. Additional copies of $i s d E F$ are also present in BB2000. Its role in selfrecognition as an orphan genes is speculative [9]. The comparison analysis of BB2000 and K1609 strains shown 99-100\% of homology between $i d s A B C D E F$ genes. However, the $i d s D$ gene possesses a fragment of low homology between K1609 and BB2000 in the central part. Strains K1609 and BB2000 demonstrate homology in ids operon organization (Fig. 4a), which corresponds to their genomic similarities.

Both K1609 and K670 strains possess the idrABCD genes, whereas the $i d r E$ was found only in the strain K670. In both genomes $i d r A$ is located at a considerable distance from the $i d r B C D E$ cluster. The $i d r D$ gene from BB2000, potentially encoding the toxic protein [4], share $99 \%$ and 98\% identity with K1609 and K670, respectively (Fig. 4b). Using BLAST we were not able to detect significant homology to gene encoding the PefD toxin of pef operon presented in HI4320 strain [5]. Differences in $i d s$ and $i d r$ operon between K1609 and K670 might be the molecular factor responsible for the strains recognition as non-kin.

The overexpression of $r s b A$ gene contributes to the precocious phenotype in $P$. mirabilis that is characterized by defect in the temporal control of swarming migration. Such strains start swarming ca. 60 min. earlier [28]. After annotation, we observed differences in sequence of $r s b A$ between K1609 and K670. In both strains, this polymorphism did not contribute to the amino acid sequence of RsbA protein, comparing to BB2000. Nevertheless, it cannot be rejected that these silent mutations do not contribute to the RsbA function most likely by a distorted balance of the protein folding process [29]. Next we identified a single point mutation in $r c s C$ gene in $\mathrm{K} 670$ genome. The mutation results in serine presented in BB2000 and K1609 at 873 position substitution with the arginine. The mutation occurred in the region of receiver domain in $\mathrm{Rcs} C$ protein [30]. The $\mathrm{RcsB}$ and $\mathrm{RcsC}$ are members of a two-component regulatory circuit controlling capsular synthesis, where $\mathrm{Rcs} C$ is a histidine kinase and $\mathrm{RcsB}$ is its cognate response regulator. The $\operatorname{rcs} B$ and $r c s C$ are located in P. mirabilis downstream the $r s b A$. It was shown that distribution of $r c s C$ gene in $P$. mirabilis BB2000 results in similar precocious phenotype as in case of $r s b A$ [28]. In both studied strains, we did not detect any missense mutation in $r c s B$ gene.

\section{Conclusions}

Within presented genome announcement, we report draft genome sequences of two $P$. mirabilis strains that exhibit differences in terms of territoriality advantages. We 


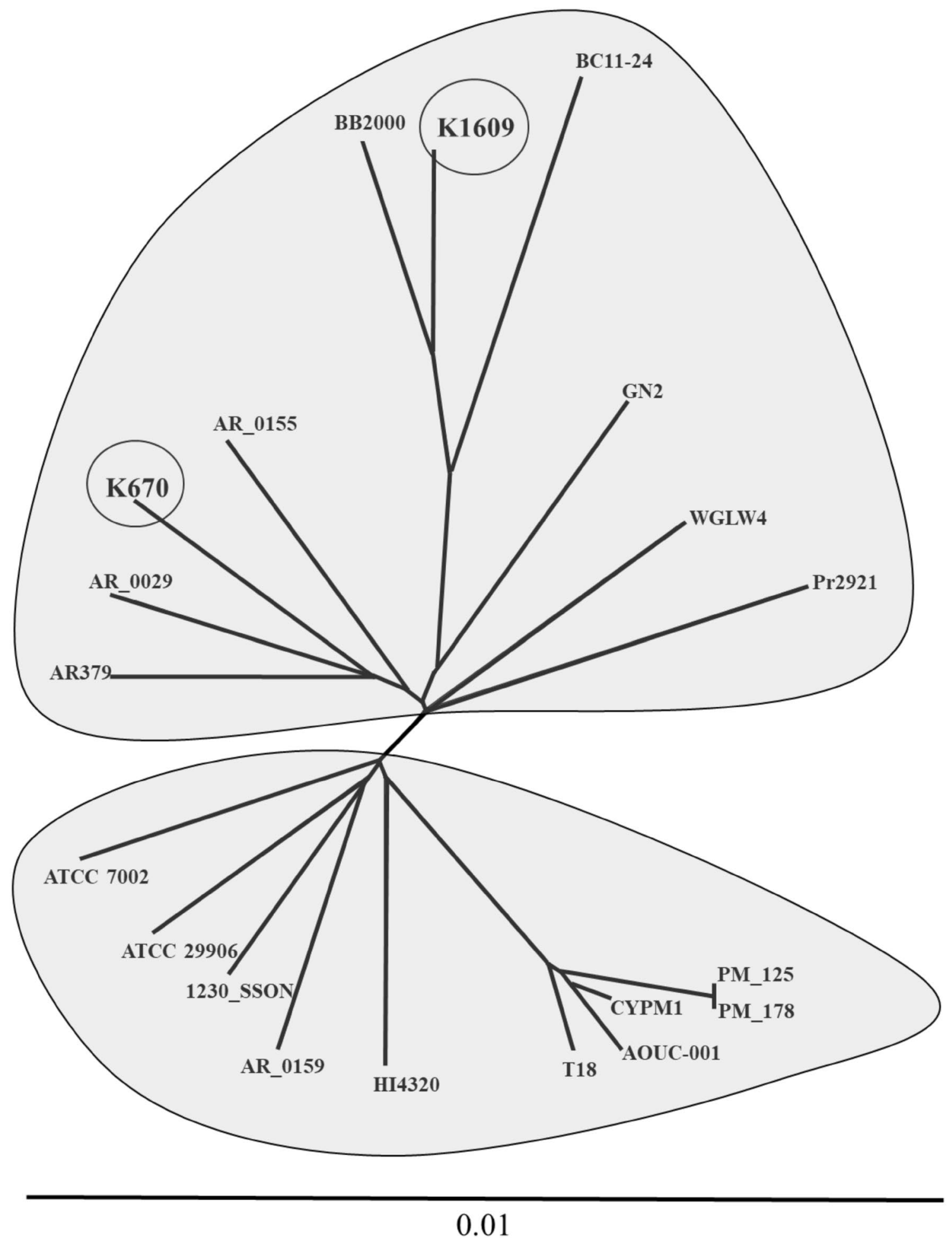

Fig. 2 Neighbor-joining tree of Proteus mirabilis K1609 and K670 and closely related P. mirabilis strains based on whole-genome ANI distance matrix

hypothesize the possible role of differences within $r s c C$ and self-recognition genes in swarming initiation control and recognition of kin, respectively. Our in silico analysis provided basic genomic insight that will serve for further examination of the self-recognition and territoriality in $P$. mirabilis K1609 and K670 model system. The P. mirabilis 


\section{A}

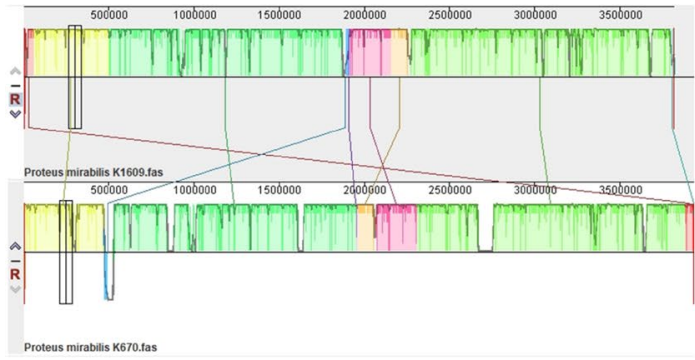

B

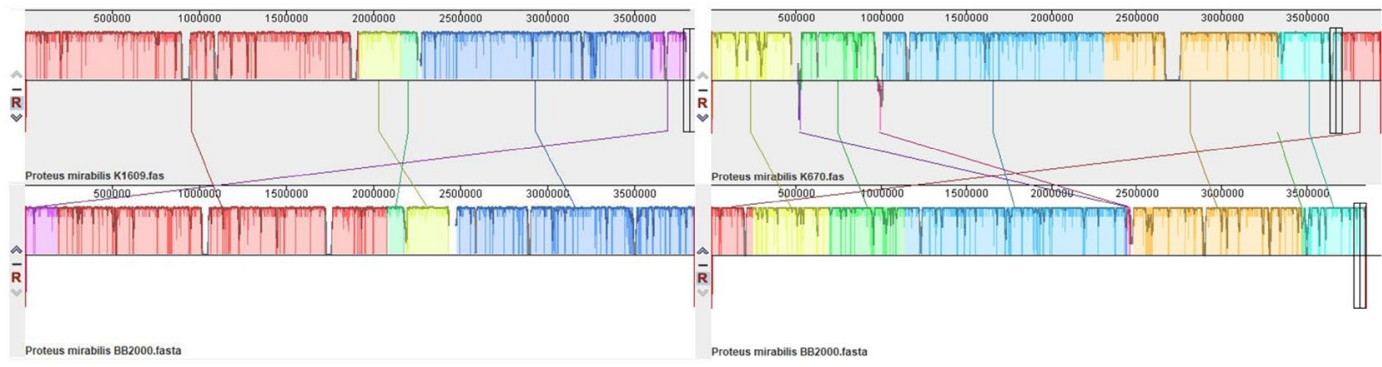

C

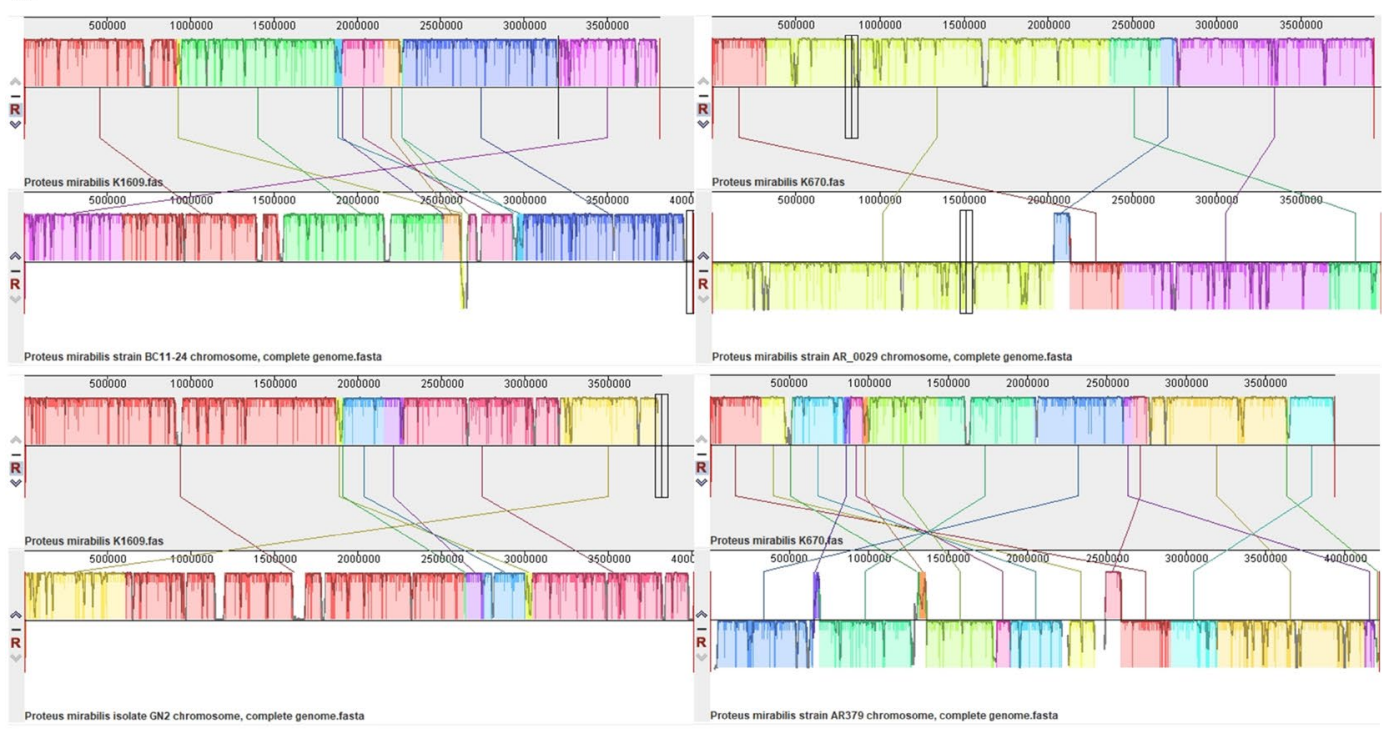

D

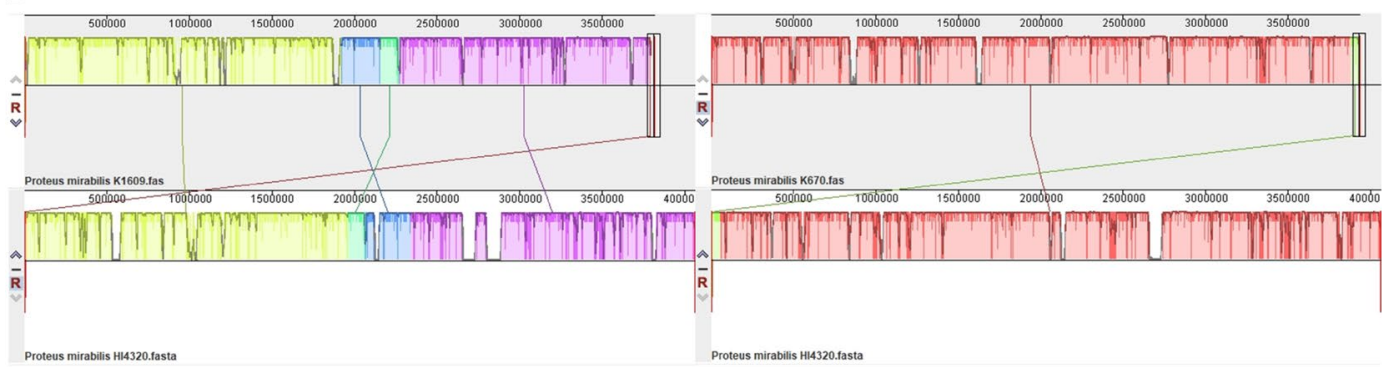

Fig. 3 Mauve comparison of Proteus mirabilis genomes. Pair-wise Whole Genome Alignment of a $P$. mirabilis strain K1609 against K670, b K1609 and K670 against BB2000, c K1609 against close rel-

atives BC11-24 and GN2 and K670 against close relatives AR_0029 and AR379, d K1609 and K670 against HI4320 

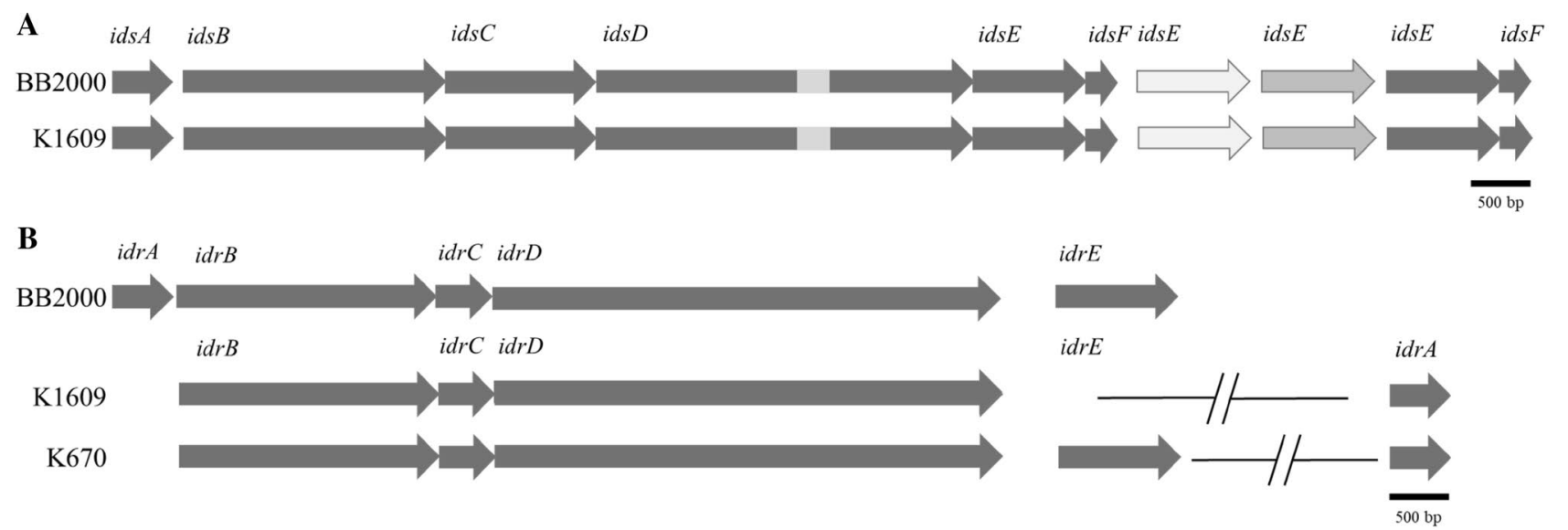

Fig. 4 Comparison of $\mathbf{a} i d s A B C D E F$ and $\mathbf{b} i d r A B C D E$ genes between Proteus mirabilis BB2000, K1609 and K670 strains. For Panel a gray scale indicates the level of genes homology. The region of low simi-

genome sequences obtained in this work were deposited at GenBank and are available under the Accession Numbers CP028522 and CP028356, for K1609 and K670, respectively.

Acknowledgements NGS sequencing was founded from the Specific Scientific Equipment Programme (Decision No. 8636/E-342/ SPUB/2016/2) allocated to I. Swiecicka. MiSeq machine is equipment of the Center of Synthesis and Analysis BioNanoTechno of University of Bialystok founded by the European Union as a part of the Operational Program Development of Eastern Poland 2007-2013 (Project POPW.01.03.00-20-034/09-00). The funders had no role in study design, data collection and analysis, decision to publish, or preparation of the manuscript. This work was partially supported by BS UJK Grant No. 612529.

Open Access This article is distributed under the terms of the Creative Commons Attribution 4.0 International License (http://creativeco mmons.org/licenses/by/4.0/), which permits unrestricted use, distribution, and reproduction in any medium, provided you give appropriate credit to the original author(s) and the source, provide a link to the Creative Commons license, and indicate if changes were made.

\section{References}

1. Jones BV, Young R, Mahenthiralingam E, Stickler DJ (2004) Ultrastructure of Proteus mirabilis swarmer cell rafts and role of swarming in catheter-associated urinary tract infection. Infect Immun 72:3941-3950

2. Gibbs KA, Greenberg EP (2011) Territoriality in Proteus: advertisement and aggression. Chem Rev 111:188-194

3. Alteri CJ, Himpsl SD, Pickens SR et al (2013) Multicellular bacteria deploy the type VI secretion system to preemptively strike neighboring cells. PLoS Pathog 9(9):e1003608

4. Wenren LM, Sullivan NL, Cardarelli L (2013) Two independent pathways for self-recognition in Proteus mirabilis are linked by type VI-dependent export. MBio 4:1-10 larity in $i d s D$ between BB2000 and K1609 is marked with light gray. For Panel b slanted lines indicate a break in the genomic regions, corresponding to approximately $2 \mathrm{Mbp}$

5. Alteri CJ, Himpsl SD, Zhu K et al (2017) Subtle variation within conserved effector operon gene products contributes to T6SSmediated killing and immunity. PLoS Pathog 13(11):e1006729

6. Pearson MM, Sebaihia M, Churcher C et al (2008) Complete genome sequence of uropathogenic Proteus mirabilis, a master of both adherence and motility. J Bacteriol 190:4027-4037

7. Sullivan NL, Septer AN, Fields AT et al (2013) The complete genome sequence of Proteus mirabilis strain BB2000 reveals differences from the $P$. mirabilis reference strain. Genome Announc 1:e00024-e00013

8. Saak CC, Zepeda-Rivera MA, Gibbs KA (2017) A single point mutation in a TssB/VipA homolog disrupts sheath formation in the type VI secretion system of Proteus mirabilis. PLoS ONE 12(9):e0184797

9. Gibbs KA, Urbanowski ML, Greenberg EP (2008) Genetic determinants of self identity and social recognition in bacteria. Science 321(5886):256-259

10. Czerwonka G, Guzy A, Kałuża K et al (2016) The role of Proteus mirabilis cell wall features in biofilm formation. Arch Microbiol. https://doi.org/10.1007/s00203-016-1249-x

11. Li B, Feng J, Zhan Z et al (2018) Dissemination of KPC-2-encoding IncX6 plasmids among multiple enterobacteriaceae species in a single Chinese hospital. Front Microbiol 9:478

12. Roach DJ, Burton JN, Lee C et al (2015) A year of infection in the intensive care unit: prospective whole genome sequencing of bacterial clinical isolates reveals cryptic transmissions and novel microbiota. PLoS Genet 11(7):e1005413

13. Pilato V, Di Chiarelli A, Boinett CJ et al (2016) Complete genome sequence of the first KPC-type carbapenemase-positive Proteus mirabilis strain from a bloodstream infection. Genome Announc 4:e00607-e00616

14. Lutgring DJ, Machado M-J, Benahmed HF et al (2018) FDA-CDC antimicrobial resistance isolate bank: a publicly available resource to support research, development, and regulatory requirements. J Clin Microbiol 56:e01415-e01417

15. Minogue TD, Daligault HE, Davenport KW et al (2014) Draft genome assemblies of Proteus mirabilis ATCC 7002 and Proteus vulgaris ATCC 49132. Genome Announc 2:e01064-e01014

16. Lei C-W, Chen Y-P, Kong L-H et al (2018) PGI2 is a novel SGI1relative multidrug-resistant genomic island characterized in Proteus mirabilis. Antimicrob Agents Chemother 62:e00019-e00018 
17. Yu CY, Ang GY, Ngeow YF et al (2016) Genome sequences of two multidrug-resistant Proteus mirabilis strains harboring CTX-M-65 isolated from Malaysia. Genome Announc 4:e01301-e01316

18. Giorello FM, Romero V, Farias J et al (2016) Draft genome sequence and gene annotation of the uropathogenic bacterium Proteus mirabilis Pr2921. Genome Announc 4:e00564-e00516

19. Aziz RK, Bartels D, Best A et al (2008) The RAST Server: Rapid annotations using subsystems technology. BMC Genom 9:1-15

20. Figueras MJ, Beaz-Hidalgo R, Hossain MJ, Liles MR (2014) Taxonomic affiliation of new genomes should be verified using average nucleotide identity and multilocus phylogenetic analysis. Genome Announc 2:e00927-e00914

21. Saitou N, Nei M (1987) The Neighbor-joining method: a new method for reconstructing phylogenetic trees. Mol Biol Evol 4:406-425

22. Zhang Z, Schwartz S, Wagner L, Miller W (2000) A greedy algorithm for aligning DNA sequences. J Comput Biol 7:203-214

23. Darling ACE, Mau B, Blattner FR, Perna NT (2004) Mauve: Multiple alignment of conserved genomic sequence with rearrangements. Genome Res 14(7):1394-1403
24. Budding AE, Ingham CJ, Bitter W et al (2009) The Dienes phenomenon: competition and territoriality in swarming Proteus mirabilis. J Bacteriol 191:3892-39008

25. Saeb ATM, Al-Rubeaan KA, Abouelhoda M et al (2017) Genome sequencing and analysis of the first spontaneous nanosilver resistant bacterium Proteus mirabilis strain SCDR1. Antimicrob Resist Infect Control 6:119

26. Coulthurst SJ (2013) The Type VI secretion system - a widespread and versatile cell targeting system. Res Microbiol 164:640-654

27. Saak CC, Gibbs KA (2016) The self-identity protein IdsD is communicated between cells in swarming Proteus mirabilis colonies. J Bacteriol 198:3278-3286

28. Belas R, Schneider R, Melch M (1998) Characterization of Proteus mirabilis precocious swarming mutants: Identification of $r s b A$, encoding a regulator of swarming behavior. J Bacteriol 180:6126-6139

29. Quax TEF, Claassens NJ, Söll D, van der Oost J (2015) Codon bias as a means to fine-tune gene expression. Mol Cell 59:149-161

30. Clarke D, Joyce S, Toutain C (2002) Genetic analysis of the RcsC sensor kinase from Escherichia coli K-12. J Bacteriol 184:1204-1208 